\title{
Olive oil inhibits DMH-induced colon tumor growth by triggering mitochondrial apoptotic pathway: An in vivo study
}

\author{
Nanda $\mathrm{N}^{1,2,3}$, Mahmood $\mathrm{S}^{2 *}$, Mahmood A ${ }^{4}$, Bhat M Owais ${ }^{5}$ and Dhawan $\mathrm{DK}^{3 *}$ \\ ${ }^{1}$ Department of Pathology, Sol Goldman Pancreatic Cancer Research Center, Johns Hopkins Medical Institutions, Baltimore, Maryland, USA \\ ${ }^{2}$ Department of Experimental Medicine and Biotechnology, Postgraduate Institute of Medical Education and Research (PGIMER), Chandigarh, India \\ ${ }^{3}$ Department of Biophysics, Panjab University, Chandigarh, India \\ ${ }^{4}$ Department of Biochemistry, Panjab University, Chandigarh, India \\ ${ }^{5}$ Department of Pharmacology and Toxicology, Virginia Commonwealth University, School of Medicine, Richmond, VA, USA
}

\begin{abstract}
Olive oil, a rich source of antioxidants, exhibits anti-inflammatory and anti-tumorigenic properties; however, the role of olive oil has not been systematically evaluated in the preclinical model of colon cancer. In this study, we investigated the chemopreventive role of olive oil on animal model of colon carcinogenesis by targeting its apoptotic machinery. In this study, DMH was used to induce colon cancer in rats; while olive oil was given to separate group of rats together with DMH treatment. Potent pro-apoptotic activity of olive oil was explored with apoptotic assays by FACS and confocal microscopy. It was observed that olive oil administration directly controls necrosis, and positively regulates apoptosis as assessed by Annexin V staining. Furthermore, ultrastructure examination by transmission electron microscopy (TEM) revealed that olive oil treatment ameliorates the mitochondrial damage in rat colon which was degenerated upon DMH treatment. Thus, these findings suggest that olive oil administration has a protective role in colon carcinogenesis and emphasizes new insights into chemoprevention by targeting mitochondrial/ intrinsic cell death pathway.
\end{abstract}

\section{Introduction}

Colorectal cancer (CRC) is the third most common cancer and a major cause of morbidity and mortality. About 1.2 million new cases of colorectal cancer are diagnosed each year and more than 60000 die due to this disease $[1,2]$. The continuing enormity of the colon cancer indicates that new approaches to the control of this malignant neoplastic disease are essentially required. Chemoprevention is a better approach to control incipient malignancy by regulating numerous intracellularsignaling cascades. Epidemiological studies links Mediterranean diet and cancer chemoprevention, revealed that the dietary factors significantly influence cancer risk in humans $[3,4]$.

Olive oil is a key component of the Mediterranean diet and is believed to have numerous beneficial effects due to its antioxidant properties [4]. Olive oil is a chemopreventive phytochemical that can block the initiation process or reverse the promotion stage of multistep carcinogenesis. Several in vitro studies suggest that, in addition to antioxidant ability, olive oil phenols exert anti-inflammatory and antineoplastic activities against cancer cells [5]. In contrast, there are few reports in the literature that describe the effect of olive oil in cancer prevention and its underlying mechanisms, particularly in animal model of colon carcinogenesis. Therefore, additional investigations are required to evaluate the role of olive oil in colon cancer prevention, especially under in vivo conditions.

Thus, the present study was undertaken to investigate the potential chemopreventive efficacy of olive oil in rats subjected to DMHinduced colon cancer. It was observed that olive oil administration prevented tumor formation in the colon; the prevention is associated with enhancement of apoptosis and reduction of necrosis. The study also explored the intrinsic/mitochondria-mediated apoptotic cell death pathway by examining the ultrastructure integrity of mitochondria to corroborate the potential chemopreventive effects of olive oil in colon carcinogenesis.

\section{Materials and methods}

\section{Olive oil}

Olive oil was purchased from company Figarro, Deoleo S.A., Spain. The nutrition facts of olive oil per 100g were: Energy 900Kcal, Protein 0g, Total carbohydrate $0 \mathrm{~g}$, Total fat $100 \mathrm{~g}$ (Saturated fat $13 \mathrm{~g}$, Monosaturated fat $79 \mathrm{~g}$, Polyunsaturated fat $8 \mathrm{~g}$, Trans fat $0 \mathrm{~g}$ ), Cholesterol $0 \mathrm{mg}$ and Sodium 0mg.

\section{Animals and experimental design}

All animal studies were performed in accordance with protocols approved by the Institutional Animal Ethics Committee of PGIMER,

${ }^{\star}$ Correspondence to: Devinder Kumar Dhawan, Professor, Department of Biophysics, Panjab University, Chandigarh-160014, India, E-mail: dhawan@pu.ac.in

Safrun Mahmood, Geneticist, Department of Experimental Medicine and Biotechnology, Research Block-B, Postgraduate Institute of Medical Education and Research (PGIMER), Chandigarh, India, E-mail: mahmoodpgi@gmail.com

Key words: chemoprevention, colon cancer, olive oil, apoptosis, in vivo

Received: March 28, 2018; Accepted: April 13, 2018; Published: April 17, 2018 
Chandigarh. Healthy male Sprague Dawley rats weighing between 180 to $200 \mathrm{~g}$ were divided into three groups having 24 rats in each group. Rats in Group I served as the normal control and received weekly subcutaneous (s.c.) injections of normal saline. Group II rats were injected subcutaneously with freshly prepared 1,2 dimethylhydrazine $(\mathrm{DMH})$ in normal saline, weekly at a dosage of $30 \mathrm{mg} / \mathrm{kg}$ body weight, for two different time durations of 10 and 20 weeks. Rats belonging to Group III were treated with Olive oil at a dose of $1 \mathrm{~g} / \mathrm{kg}$ body weight thrice a week through oral gavaging in addition to DMH treatment as was given to group II animals (Figure 1).

\section{Transmission electron microscopy (TEM)}

Tissues after removal from the rats were fixed immediately in $3 \%$ glutaraldehyde prepared in sorenson phosphate buffer for 24 hours, and subsequently were washed in Miellonings buffer and postfixed in $1 \%$ osmium tetroxide. The samples were then dehydrated at room temperature by keeping them in different grades of $100 \%, 90 \%$, $70 \%$ and $50 \%$ ethanol for 3 minutes and three times, then infiltered. Subsequently sections were embedded in 1:1 Propylene oxide/Epon resin and were then placed in an oven and allowed to polymerize at $60^{\circ} \mathrm{C}$ for at least $48 \mathrm{~h}$. Semi-thin sections of thickness about $1 \mu \mathrm{m}$ were cut and stained with $0.5 \%$ toludine blue prepared in $1 \%$ borax solution. Further, ultrathin sections of required area were cut by using an ultramicrotome and loaded on fine copper grids of 100-300 mesh size. The sections were double stained with uranyl acetate and lead citrate. These stained sections were finally viewed under Transmission Electron Microscope (Hitachi (H-7500) Chiyoda, Tokyo, Japan), operating at 40$120 \mathrm{kV}[6]$.

\section{Apoptosis assay}

Confocal microscopy: The colonic tissues were cut into $10 \mu \mathrm{m}$ sections by using cryostat. Thereafter, the sections were washed with PBS buffer, incubated for 15 min with AnxV-FITC, and washed with Annexin binding buffer, and then incubated with Propidium iodide by using Annexin V-FITC Apoptosis Detection kit (Calbiochem). The
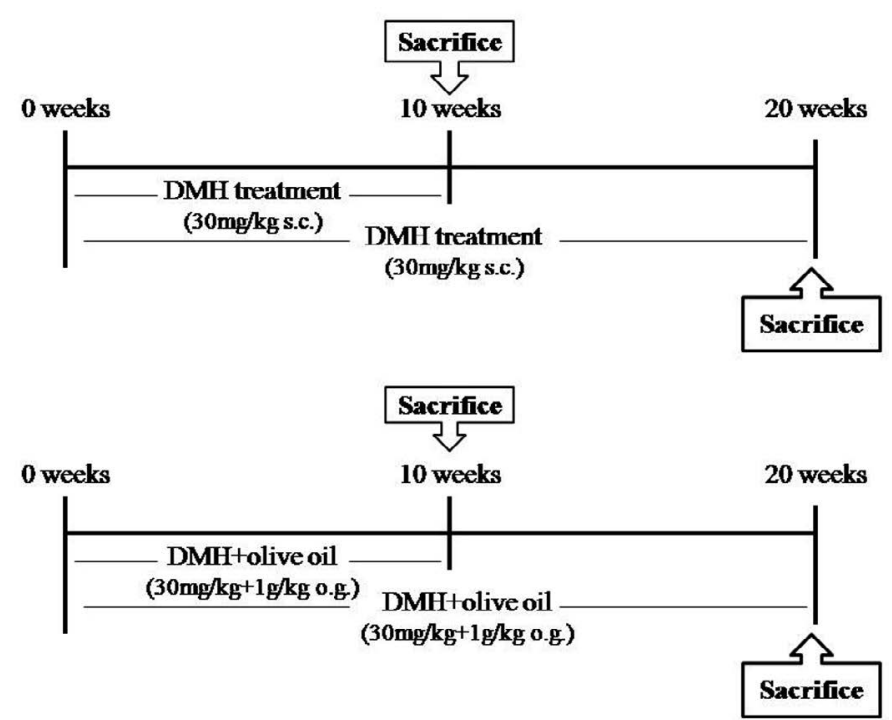

Figure 1. Olive oil treatment induces apoptosis and decreases necrosis. Apoptosis/Necrosis detection in control and treated rats by Confocal microscopy. Annexin V-FITC/PI staining was used to observe the induction of apoptosis. Annexin V stained the membrane of apoptotic cells (green fluorescence), Propidium iodide (PI) stained the nucleoli of necrotic or dead cells (red fluorescence) and merged of Annexin V-FITC/PI stained the late apoptotic cells (dual channel fluorescence). Magnification $\times 200$ sections were mounted with anti-fading agent and examined under a confocal microscope (Olympus 1x81) using standard settings for FITC fluorescence-detection [7].

Flow cytometry: For flow cytometry, colonic tissues were dissected and minced to small clumps, followed by enzymatic dissociation with $0.5 \mathrm{mg}$ collagenase IV (Sigma) in PBS for $25 \mathrm{~min}$ at $37^{\circ} \mathrm{C}$ on a shaker and centrifuged at $500 \mathrm{xg}$ for $10 \mathrm{~min}$. The supernatant containing debris was discarded; the pelleted cells were washed twice with PBS and filtered through a $100 \mu \mathrm{m}$ cell strainer to obtain single-cell suspension. Dissociated colonic cells were finally resuspended in PBS. Furthermore, cells were used for apoptotic assays using Annexin V-FITC Apoptosis Detection kit (Calbiochem) according to manufacturer's instructions. Cell sorting was performed on a BD FACS Aria II instrument in the Flow Cytometry [7].

\section{Results}

\section{Olive oil treatment stimulates apoptosis and prevents necrosis}

To examine, if olive oil, modulates the apoptotic pathway, we examined the apoptotic rate of colonic cells by using annexin $\mathrm{V}$ staining. The results were demonstrated by confocal microscopy and further validated by FACS. Interestingly, the results revealed more necrosis and less apoptosis with DMH treatment as compared to normal animals. However, olive oil treatment caused an appreciable decrease in necrosis and an increase in early and late apoptosis in DMH-treated rats.

\section{Confocal microscopy}

Based on the principle of this technique, the normal cells were not stained by the two dyes (Annexin V FITC-/PI-); the early apoptotic cells could only be stained by Annexin V-FITC (Annexin V-FITC+/ PI-); the necrotic cells were only stained with PI (Annexin V-FITC-/ $\mathrm{PI}+$ ); the late apoptotic cells were positive in both Annexin V-FITC and PI staining (Annexin V-FITC+/PI+). AnxV-FITC (green) and PI (red) stains were applied to cryostat sections of colonic tissues of all the treated and normal control rats. As shown in (Figure 2). control cells were more stained with AnxV-FITC, indicating the higher apoptotic rate in the normal control group. In the colonic sections treated with DMH alone for 10 or 20 wks, we observed less stain by Annexin

V-FITC (green), indicating that apoptotic rate was much less in colonic tissues of DMH alone treated rats. Also, we observed more PI stain (red) which confirmed that more cells were in the necrotic stage, after DMH alone treatment. However, colonic sections of DMH+olive oil treated rats at 10 or 20 weeks indicated that most cells were stained by Annexin V-FITC (green), but less by PI, indicating that most of the olive oil -treated cells were in the early apoptotic stage. Also, some other cells were stained by both the dyes, demonstrating that these cells were in the late apoptotic stage in these tissues (Figure 2).

\section{FACS analysis}

Similarly, these results also revealed that control cells were more stained with Annexin-V-positive (7.4\%), Annexin-V/PI-positive (18.1\%) and less stained with PI-positive (14.4\%), indicating the higher apoptotic rate in the normal control group. However, there were very few Annexin-V-positive (3.1\%), AnnexinV/PI-positive (7.45\%) and more PI-positive cells (22.8\%) in the DMH-treated group at 10 weeks. However, less than $20 \%$ PI positive cells were observed in olive oil treated group. While greater than $25 \%$ positive cells were noted for both Annexin V-FITC/PI staining at 10 weeks with olive oil treatment. 
Annexin V-FITC/PI staining was also completed for the 20 weeks DMH-alone and DMH +olive-treated cells. $0.4 \%$ and $21.55 \%$ annexinV positive cells; $48.4 \%$ and $9.9 \%$ PI positive cells observed for DMHalone and $\mathrm{DMH}+$ olive oil-treated cells at 20 weeks, respectively. While greater than $40 \%$ positive cells were noted for both Annexin V-FITC/ PI staining at 20 weeks with olive oil treatment as compared to DMHalone treatment (Figure 3).

\section{Olive oil restores the mitochondrial integrity in colon cancer}

Mitochondria is a central player controlling cell death, triggers the activation of caspase cascade, leading to intrinsic/mitochondrialmediated apoptotic pathway. Thus, in the present study, we also examined the viability and morphological changes in the mitochondria from colonic tissues of treated and normal control rats under transmission electron microscope. Ultrastructure examination of colonic sections of normal controls, revealed good preservation of mitochondrial morphology and integrity, while rats treated with $\mathrm{DMH}$-alone at 10 or 20 weeks exhibited mitochondrial damage with alteration in size, disappeared matrix, disorganized cristae and in some mitochondria only the outer membrane remained. However, at 10 or 20 weeks, olive oil administration protected the mitochondria by reducing the mitochondrial injury and restoring its integrity (Figure 4).

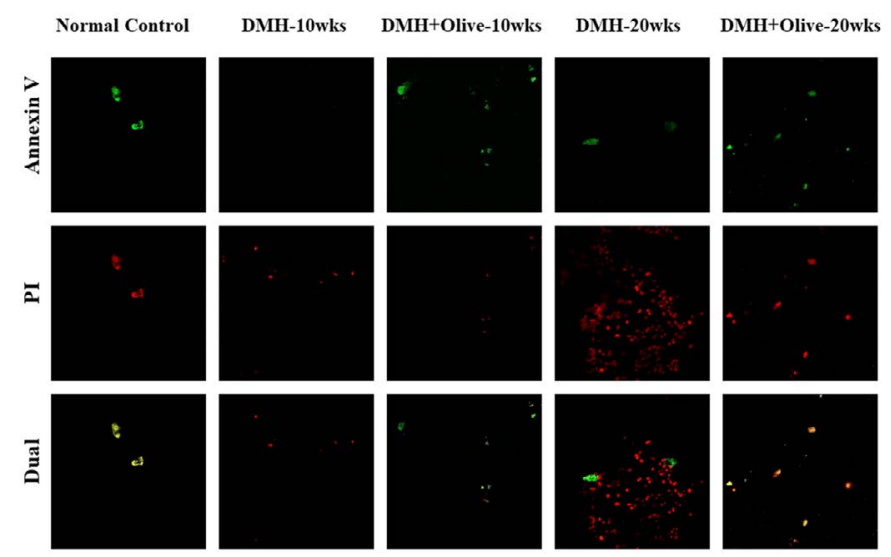

Figure 2. Apoptosis/Necrosis detection by FACS analysis. The induction of apoptosis was determined by flow cytometric analysis of Annexin V-FITC and PI-staining. Cells in the lower right quadrant (Q4) indicate Annexin V-positive (early apoptotic), upper right quadrant (Q2) indicate Annexin V-positive/PI-positive (late apoptotic), upper left quadrant (Q1) indicate PI-positive (necrotic/dead), and lower left quadrant (Q3) indicate live cells
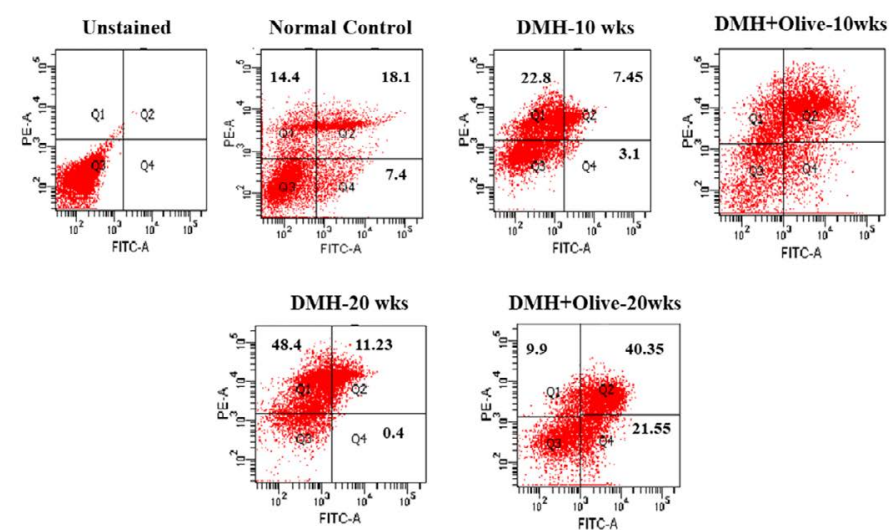

Figure 3. Effect of olive oil on mitocondria at ultrastructural level using transmission electron microscopy. Electron micrograph of normal control and treated segments of colon, Scale bar $500 \mathrm{~nm}$

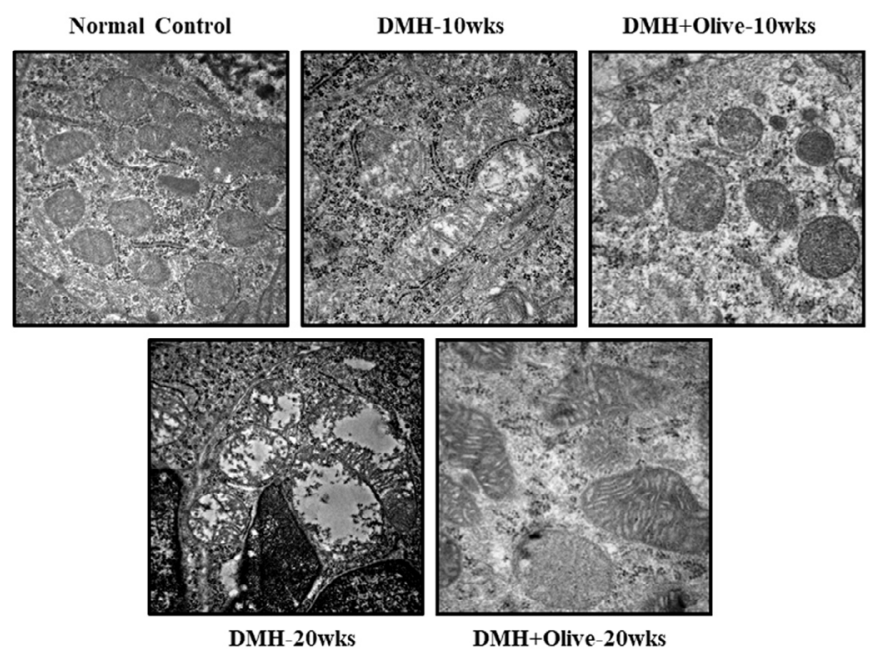

Figure 4. Olive oil administration protected the mitochondria by reducing the mitochondrial injury and restoring its integrity

\section{Discussion}

Despite persistent focus on early detection and treatment of cancer, many develop disease due to less prominence on prevention. Thus, in the present study, with more emphasis on prevention, role of olive oil and its potential has been successfully elucidated in the CRC pathogenesis with a special focus on the molecular basis of its heterogeneity. It is now very well evident that dietary agents inhibit tumor growth by targeting different machanisms involved in the process of carcinogenesis that further leads to the induction of apoptosis [8]. Similarly, we also confirmed the direct effect of olive oil on the rate of apoptosis/necrosis that showed a decrease in necrosis and increased early/late apoptosis in comparison to $\mathrm{DMH}$ treatment as shown by annexin $\mathrm{V}$ staining. It is believed that necrosis might be considered as a prognostic factor of colorectal cancer, but the underlying mechanism remains unclear [9-11]. Since TNF (tumor necrosis factor) is secreted by inflammatory cells, may be involved in inducing necrosis and stimulates tumor promotion, thus, the plausible reason for this may be TNF- $\alpha$-induced NF- $\mathrm{KB}$ activation resulted in an inflammation mediated carcinogenesis [12-14]. However, in cancer, TNF is a double-edged sword which could be either pro- or anti-tumorigenic. On one hand, TNF could be an endogenous tumor promoter, because TNF stimulates the tumor promotion, angiogenesis, invasion and metastasis of cancer cells via its molecular targets NF- $\kappa B$, VEGF and MMPs [15-18]. On the other hand, TNF could be a cancer killer by JNK activation, an important contributor to death response via inhibition of NF- $\kappa B$ activation which further increases susceptibility to TNF-induced death [19]. JNK activation is required for cytochrome $c$ release and further activation of caspase cascade to induce apoptosis [20]. Pham et al., (2004) demonstrated that activation of NF- $\kappa B$ inhibits TNF- $\alpha$-induced apoptosis by controlling the activation of JNK cascade. Thus, the present results clearly demonstrated that olive oil may regulates apoptosis/necrosis by activation of caspase cascade and inhibition of TNF- $\alpha$-induced NF- $\mathrm{kB}$ pathway. Our findings gain strength from the study of [21], who elucidated two mechanisms of apoptosis either by $\mathrm{p} 53$ mediated mitochondrial pathway that leads to the activation of caspase- 9 which in turn activates caspase-3 [22] or by NF- $\kappa \mathrm{B}-$ mediated pathway. This supports the present findings involving the chemopreventive role of olive oil in this process.

After observing appreciable effect of olive oil on rate of apoptosis and necrosis, we assumed that olive oil induced apoptosis is supposed to 
be due to the activation of mitochondrial/intrinsic apoptotic pathway. Thus, we desired to observe the effect of olive oil on mitochondrial morphology to substantiate the above presumption. In the present study, the viability and morphological changes in the mitochondria from colonic tissues were determined by transmission electron microscopy.

Mitochondrial dynamics have roles beyond maintenance of morphology, had impact on cell death, and its alterations are important for inflammatory bowel disease, colitis and colon cancer patients [2325] observed the mitochondria hyperplasia and absence of goblet cells in neoplastic glands of colonic tissue in $\mathrm{DMH}$-induced rat model. Other studies have shown that mitochondrial numbers are varied in DMH-treated animals as well as human biopsies of colon cancer as compared to control counterparts [26,27]. Likewise, we also observed the mitochondrial alterations in $\mathrm{DMH}$-treated rat colon, while it has been demonstrated that olive oil treatment ameliorates the mitochondrial damage in rat colon that was degenerated upon $\mathrm{DMH}$ treatment [28-30] As of now, no information is available with regard to ultrastructure examination of colonic tissues inflicted with DMH injury and co-treatment with olive oil. Since, [24] showed that mitochondria are dynamic structures that undergo fusion and fission process continuously throughout the life of a cell, possibly in present study, olive oil keep the balance between fission-fusion process of mitochondria to maintain its functional level. However, further studies may be needed to explore the dynamic structure of mitochondria after olive oil treatment, that may further explain the chemopreventive role of olive oil in colon carcinogenesis.

\section{Conclusion}

The present study revealed the potential role of olive oil in preventing the initiation and development of colon carcinogenesis in experimental animals. The mode of action of olive oil may involve activation of mitochondrial/intrinsic apoptotic pathway characterized by alteration of mitochondrial structure and rate of apoptosis, which may have associated with the suppression of inflammatory pathway. Thus, the current findings might build a persuasive case for chemopreventive role of olive oil in targeting the different stages of colon carcinogenesis and open up a new vista for future research to be translated to human subjects to control incipient malignancy.

\section{Acknowledgement}

We thank Mr Sunil Kumar for helping to carry out the animal work and Ms Rekha verli for her technical assistance.

\section{Declarations of interest}

We thank Indian Council of Medical Research (ICMR), New Delhi, India, for financial support to the research work. All the authors have no conflict of interest.

\section{Reference}

1. American Cancer Society (2014) Colorectal Cancer Facts \& Figures 2014-2016. Atlanta: American Cancer Society, USA.

2. Siegel R, Desantis C, Jemal A. (2014) Colorectal cancer statistics, 2014. CA Cancer J Clin 64: 104-117. [Crossref]

3. Serra-Majem L, Roman B, Estruch R (2006) Scientific evidence of interventions using the Mediterranean diet: a systematic review. Nutr Rev 64: S27-S47. [Crossref]

4. Verberne L, Bach-Faig A, Buckland G et al. (2010) Association between the Mediterranean diet and cancer risk: a review of observational studies. Nutr Cancer 62: 860-870. [Crossref]
5. Sofi F, Cesari F, Abbate R et al. (2008) Adherence to Mediterranean diet and health status: meta-analysis. BMJ 337: 1344.

6. Willett WC, Sacks F, Trichopoulou A et al. (1995) Mediterranean diet pyramid: a cultural model for healthy eating. Am J Clin Nutr 61: 1402-1406. [Crossref]

7. Casaburi I, Puoci F, Chimento A (2013) Potential of olive oil phenols as chemopreventive and therapeutic agents against cancer: a review of in vitro studies. Mol Nutr Food Res 57: 71-73.

8. Singla N, Dhawan DK. (2015) Zinc down regulates Apaf-1-dependent Bax/Bcl-2 mediated caspases activation during aluminium induced neurotoxicity. Biometals 28 : 61-73. [Crossref]

9. Weigmann B, Tubbe I, Seidel D, Nicolaev A, Becker C, Neurath MF. (2007) Isolation and subsequent analysis of murine lamina propria mononuclear cells from colonic tissue. Nat Protoc 2: 2307-2311. [Crossref]

10. Khan N, Afaq F, Mukhtar H (2007) Apoptosis by dietary factors: the suicide solution for delaying cancer growth. Carcinogenesis 28: 233-239. [Crossref]

11. Pollheimer MJ, Kornprat P, Lindtner RA (2010) Tumor necrosis is a new promising prognostic factor in colorectal cancer. Hum Pathol 41: 1749-1457. [Crossref]

12. Richards CH, Roxburgh CS, Anderson JH (2012) Prognostic value of tumour necrosis and host inflammatory responses in colorectal cancer. BrJ Surg 99: 287-294. [Crossref]

13. Komori K, Kanemitsu Y, Kimura K (2013) Tumor necrosis in patients with TNM stage IV colorectal cancer without residual disease (R0 Status) is associated with a poor prognosis. Anticancer Res 33: 1099-1105. [Crossref]

14. Yang H, Bocchetta M, Kroczynska B (2006) TNF-alpha inhibits asbestos-induced cytotoxicity via a NF-kappaB-dependent pathway, a possible mechanism for asbestosinduced oncogenesis. Proc Natl Acad Sci U S A 103: 10397-10402. [Crossref]

15. Cooks T, Pateras IS, Tarcic O (2013) Mutant p53 prolongs NF- $\kappa B$ activation and promotes chronic inflammation and inflammation-associated colorectal cancer. Cancer Cell 23: 634-646. [Crossref]

16. Ardestani S, Deskins DL, Young PP (2013) Membrane TNF-alpha-activated programmed necrosis is mediated by Ceramide-induced reactive oxygen species. $J \mathrm{Mol}$ Signal 8: 12. [Crossref]

17. Suzukawa K, Weber TJ, Colburn NH (2002) AP-1, NF-kappa-B, and ERK activation thresholds for promotion of neoplastic transformation in the mouse epidermal JB6 model. Environ Health Perspect 110: 865-870. [Crossref]

18. Hu J, Nakano H, Sakurai H (2004) Insufficient p65 phosphorylation at S536 specifically contributes to the lack of NF-kappaB activation and transformation in resistant JB6 cells. Carcinogenesis 25: 1991-2003. [Crossref]

19. Kulbe H, Thompson R, Wilson JL (2007) The inflammatory cytokine tumor necrosis factor-alpha generates an autocrine tumor-promoting network in epithelial ovarian cancer cells. Cancer Res 67: 585-592. [Crossref]

20. Esteve PO, Chicoine E, Robledo O (2002) Protein kinase C-zeta regulates transcription of the matrixmetalloproteinase-9 gene induced by IL-1 and TNF-alpha in glioma cells via NF-kappa B. J Biol Chem 277: 35150-35155. [Crossref]

21. Mon NN, Hasegawa H, Thant AA (2006) A role for focal adhesion kinase signaling in tumor necrosis factor-alpha-dependent matrix metalloproteinase-9 production in a cholangiocarcinoma cell line, CCKS1. Cancer Res 66: 6778-6784. [Crossref]

22. Kamata H, Honda S, Maeda S (2005) Reactive oxygen species promote TNF alphainduced death and sustained JNK activation by inhibiting MAP kinase phosphatases. Cell 120: 649-661. [Crossref]

23. Wang S, Liu Z, Wang L (2009) NF-kappaB signaling pathway, inflammation and colorectal cancer. Cell Mol Immunol 6: 327-334. [Crossref]

24. Khan R, Khan AQ, Lateef A (2013) Glycyrrhizic acid suppresses the development of precancerous lesions via regulating the hyperproliferation, inflammation, angiogenesis and apoptosis in the colon of Wistar rats. PLoS One 8: 56020. [Crossref]

25. Jin S, Levine AJ (2001) The p53 functional circuit. J Cell Sci 114: 4139-4140. [Crossref]

26. Chen H, Chan DC (2005) Emerging functions of mammalian mitochondrial fusion and fission. Hum Mol Genet 14: 283-289. [Crossref]

27. Okamoto K, Shaw JM (2005) Mitochondrial morphology and dynamics in yeast and multicellular eukaryotes. Annu Rev Genet 39: 503-536. [Crossref]

28. Kim JC, Gong GY, Park KY (1992) Applicability of DMH-induced colorectal tumor model in respect to carcinogenesis. J Korean Cancer Assoc 24: 239-248. 
Nanda N (2018) Olive oil inhibits DMH-induced colon tumor growth by triggering mitochondrial apoptotic pathway: An in vivo study

29. Toth B, Malick L, Shimizu H (1976) Production of intestinal and other tumors by 1, 2-dimethylhydrazine dihydrochloride in mice. A light and transmission electron microscopic study of colonic neoplasms. Am J Pathol 84: 69-76. [Crossref]
30. DaCosta RS, Andersson H, Cirocco M (2005) Autofluorescence characterisation of isolated whole crypts and primary cultured human epithelial cells from normal, hyperplastic, and adenomatous colonic mucosa. J Clin Pathol 58: 766-774. [Crossref]

Copyright: $\odot 2018$ Nanda N. This is an open-access article distributed under the terms of the Creative Commons Attribution License, which permits unrestricted use, distribution, and reproduction in any medium, provided the original author and source are credited. 